\title{
Actinomicetes CUltiVABLES Asociados a MiCROECOSISTEMAS LIQUÉNIICOS de la selva templada VALdiviana, Argentina
}

\author{
JOSE M. SCERVINO*,1, MARÍA I. MESSUTI ${ }^{*, 1}$, MARIANA SOLANS ${ }^{1}$ y GERNOT VOBIS ${ }^{1}$
}

\begin{abstract}
Summary: Cultivable actinomycetes associated to lichenized microecosystems from the temperate Valdivian rainforests, Argentina. Twenty-three (23) cultivable strains of actinomycetes were isolated from the corticolous lichen species Pseudocyphellaria berberina present in the Valdivian temperate rainforest, Argentina, being twenty-one (21) endolichenic and two (2) ectolichenic. Seventeen (17) strains were identified as belonging to genera Actinoplanes (2), Dactylosporangium (1), aff. Pseudonocardia (1), Micromonospora (6), aff. Micromonospora (1) and Streptomyces (5), Streptosporangoium (1). This is the first record of actinomycetes isolated from lichenized fungi for Argentina.
\end{abstract}

Key words: Actinomycetes, Argentina, lichenized fungi, Pseudocyphellaria, Valdivian temperate rainforest.

\begin{abstract}
Resumen: Se aislaron veintitrés (23) cepas de actinomicetes cultivables de la especie corticícola Pseudocyphellaria berberina presente en la selva templada valdiviana, Argentina, veintún (21) fueron endoliquénicas y dos (2) ectoliquénicas. Se identificaron diecisiete (17) cepas de los géneros Actinoplanes (2), Dactylosporangium (1), aff. Pseudonocardia (1), Micromonospora (6), aff. Micromonospora (1), Streptomyces (5) y Streptosporangoium (1). Este es el primer registro para la Argentina de actinomicetes aislados de hongos liquenizados.
\end{abstract}

Palabras clave: Actinomicetes, Argentina, hongos liquenizados, Pseudocyphellaria, selva templada valdiviana.

\section{INTRODUCCIÓN}

En la mayoría de los ecosistemas, las comunidades microbianas presentan una amplia distribución, desarrollándose en diferentes tipos de sustratos y mostrando un papel importante en todos los ciclos biogeoquímicos (Rousk \& Bengtson, 2014). El talo de diversos grupos de criptógamas, como los hongos y las briofitas, resulta un hábitat propicio para el establecimiento y desarrollo de variadas comunidades bacterianas, incluyendo representantes de Alphaproteobacteria, Betaproteobacteria y Actinobacteria entre otras (Cardinale et al., 2006, 2008). La organización

\footnotetext{
* Ambos autores contribuyeron de igual manera a la ejecución de este trabajo.

${ }^{1}$ Departamento de Botánica, CRUB, INIBIOMA-CONICETUNComahue, Quintral 1250, 8400 S.C. de Bariloche, Río Negro, Argentina. jmscervino@hotmail.com o mimessuti@gmail.com
}

morfológica y anatómica de los hongos liquenizados constituyen un microecosistema estable para el desarrollo de consorcios de bacterias aeróbicas no fotoautotróficas como componentes integrales del talo (Grube et al., 2009; Hodkinson \& Lutzoni, 2009; Cardinale et al., 2012; Hodkinson et al., 2012). Estos consorcios incluyen a un conjunto de bacterias filamentosas Gram-positivas con alto contenido de $\mathrm{G}+\mathrm{C}$ en su $\mathrm{ADN}$ (entre 50 hasta más de un $70 \mathrm{~mol} \%$ ) correspondientes al phylum Actinobacteria, más frecuentemente referidas al grupo de los actinomicetes (Goodfellow et al., 2012); los que parecen cumplir roles vinculados con la interacción micobionte-fotobionte relacionados con el flujo de nutrientes, señales químicas y metabolitos secundarios (González et al., 2005; Mushegian et al., 2011). La anatomía del talo liquénico varía desde aquellos con una marcada diferenciación interna, incluyendo la estratificación de los simbiontes, hasta aquellos que muestran una organización homogénea (micobionte 
y fotobionte uniformemente distribuidos). De acuerdo a su ubicación en el talo, las poblaciones bacterianas pueden ser ectoliquénicas, cuando se encuentran sobre la corteza superior y/o inferior, o endoliquénicas, cuando están asociadas a las capas internas (Grube et al., 2009).

El objetivo de este trabajo fue evaluar la presencia de actinomicetes cultivables en microecosistemas liquénicos representados por especímenes de la especie Pseudocyphellaria berberina (G. Forst.) D. J. Galloway \& P. James (Ascomycota) coleccionados en el área de Puerto Blest, selva templada lluviosa valdiviana (Río Negro, Argentina).

\section{Materiales y Métodos}

\section{Área de estudio}

La región de Puerto Blest, incluida en la selva templada valdiviana, se encuentra en el extremo occidental del Brazo Blest del Lago Nahuel Huapi dentro del Parque Nacional Nahuel Huapi, Río Negro, Argentina, entre los $41^{\circ} 01^{\prime} \mathrm{S}$ y $71^{\circ} 48^{\prime} \mathrm{O}$. Desde el punto de vista fitogeográfico pertenece a la Provincia Subantártica, Distrito Valdiviano, siendo esta una de las zonas más lluviosas del país con precipitaciones que alcanzan aproximadamente los 3000-4000 mm anuales (Cabrera \& Willink, 1973).

\section{Colección de las muestras}

Mediante el método de caminata al azar se coleccionaron especímenes de hongos liquenizados foliosos epífitos utilizando un cuchillo y bolsas de papel estériles. Entre ellos se seleccionaron, para este estudio, dos ejemplares identificados como $P$. berberina provenientes de dos especies diferentes de forofitos del estrato arbóreo dominante. Uno, epífito sobre corteza de Nothofagus dombeyi (Mirb.) Oerst., denominado en este trabajo como "liquen 3" (L3), y el otro sobre corteza de Saxegothaea conspicua Lindl., denominado en este trabajo como "liquen 8" (L8). Ambos ejemplares se secaron a temperatura ambiente durante 7 días y se conservaron a $4^{\circ} \mathrm{C}$ hasta su posterior procesado.

\section{Aislamiento de actinomicetes}

Los hongos liquenizados se trataron siguiendo la técnica propuesta por Möller (1994). Una porción del talo $(1 \mathrm{~g})$ se lavó con agua estéril y posteriormente se maceró en mortero hasta obtener un material homogéneo. Para los aislamientos de los organismos ectoliquénicos, el agua de lavado se conservó a $4^{\circ} \mathrm{C}$; y para el aislamiento de los organismos endoliquénicos, el homogenato se resuspendió en $30 \mathrm{ml}$ de agua estéril y se conservó a $4^{\circ} \mathrm{C}$. Para el aislamiento de las cepas de actinomicetes se siguió el protocolo propuesto por da Silva et al. (2011) por triplicado y modificado, utilizando en cajas de Petri el medio artificial suelo-agar (KEHE) (Vobis, 2006), suplementado con extracto de liquen $1 \%(\mathrm{~V} / \mathrm{V})(\mathrm{KEHE}+\mathrm{L})$. Con el fin de aislar los actinomicetes ectoliquénicos y endoliquénicos se realizaron diluciones seriadas del agua de lavado y del homogenato respectivamente. En el medio de cultivo KEHE + L se sembró $0,1 \mathrm{ml}$ de cada una de las diluciones seriadas y se incubó cada caja a $28^{\circ} \mathrm{C}$ durante 20 días. $\mathrm{Al}$ término de este período, se realizó un recuento general de las unidades formadoras de colonias (UFC) de todos los microorganismos, y se aislaron y transfirieron aquellas posibles cepas pertenecientes al grupo de los actinomicetes. Para la identificación de estos últimos a nivel de género los aislamientos se cultivaron en el medio sólido KEHE y en medio con agar extracto de levadura y almidón (YpSs), en ambos casos a $28^{\circ} \mathrm{C}$ durante 20 días (Shirling \& Gottlieb 1966; Vobis 1997, 2006). Las cepas de actinomicetes aisladas se depositaron en el cepario del herbario BCRU (Tabla 1).

\section{Resultados y Discusión}

Las UFC $\mathrm{x} \mathrm{g}^{-1}$ determinadas fueron similares en los dos talos, L3 y L8, de P. berberina. Además, se registró un número, aproximadamente, cinco veces mayor de bacterias sobre la corteza del talo (microorganismos ectoliquénicos) que en el interior del mismo (microorganismos endoliquénicos) (Fig. 1). Al menos para este sistema de estudio, el forofito donde $P$. berberina se desarrolla no determinaría el número de bacterias presentes en el microecosistema liquénico. Esto concuerda con la hipótesis que las comunidades bacterianas asociadas a los hongos liquenizados no son meras extensiones de aquellas que se encuentran en los sustratos circundantes (Bates et al., 2011). Si bien el recuento de UFC x $\mathrm{g}^{-1}$ totales indica que, en ambos talos, la mayoría de estos organismos son ectoliquénicos (Fig. 1), se observó que en el caso particular de los actinomicetes 
Tabla 1. Características de las actinobacterias cultivables aisladas de Pseudocyphellaria berberina.

\begin{tabular}{|c|c|c|c|c|c|c|}
\hline \multicolumn{7}{|c|}{ Características } \\
\hline Cepa & Género & Ubicación & $\begin{array}{l}\text { Micelio } \\
\text { sustrato }\end{array}$ & $\begin{array}{c}\text { Micelio } \\
\text { aéreo }\end{array}$ & Pigmentos $^{s}$ & Tipo de esporulación \\
\hline MITI $1^{a}$ & N.I. & ED & anaranjado & - & - & N.O. \\
\hline MITI $1^{b}$ & aff. Micromonospora & ED & anaranjado & - & - & N.O. \\
\hline $\mathrm{MITI} 2^{\mathrm{a}}$ & Micromonospora & ED & anaranjado & rudimentario & - & individuales \\
\hline MITI $2^{\mathrm{b} 1}$ & Micromonospora & ED & anaranjado & rudimentario & - & individuales \\
\hline MITI $2^{\mathrm{b} 2}$ & Micromonospora & ED & anaranjado & rudimentario & - & individuales, en racimos \\
\hline MITI $2^{\mathrm{b} 3}$ & Micromonospora & ED & anaranjado & rudimentario & - & N.O. \\
\hline MITI 3 & Micromonospora & EC & anaranjado & rudimentario & - & individuales, en racimos \\
\hline MITI 4 & N.I. & EC & amarillo & - & - & N.O. \\
\hline MITI 5 & Streptomyces & ED & castaño & gris ceniza & ferrugíneo & en cadenas (V-S) \\
\hline MITI 6 & Actinoplanes & ED & rosado & - & - & en esporangios subglobosos \\
\hline MITI 8 & Streptosporangium & ED & rosado & blanco & - & en esporangios globosos \\
\hline MITI 9 & N.I. & ED & amarillo & - & - & individuales \\
\hline MITI 11 & Streptomyces & ED & rosado & gris ceniza & melanoide & en cadenas $(F)$ \\
\hline MITI 12 & Streptomyces & ED & rosado & - & melanoide & en cadenas (RA) \\
\hline MITI 13 & Streptomyces & ED & rosado & rosado & - & en cadenas (RA) \\
\hline MITI $14^{\mathrm{a}}$ & Micromonospora & ED & anaranjado & - & - & en esporangios globosos \\
\hline MITI 14b & Actinoplanes & ED & anaranjado & - & - & en esporangios globosos \\
\hline MITI 15 & Streptomyces & ED & rosado & blanco & - & en cadenas $(F)$ \\
\hline MITI 16 & N.I. & ED & blanco & - & - & artrosporas en agregdos \\
\hline MITI 18 & N.I. & ED & amarillo & - & - & individuales \\
\hline MITI 20 & N.I. & ED & amarillo & - & - & N.O. \\
\hline MITI 21 & Dactylosporangium & ED & anaranjado & - & - & en esporangios dactiliformes \\
\hline MITI 22 & aff. Pseudonocardia & ED & amarillo & blanco & - & N.O. \\
\hline
\end{tabular}

Referencias: s: pigmentos solubles. N.I.: No identificado. N.O.: No observado. (-): Ausente. ED: Endoliquénico. EC: Ectoliquénico. V-S: Configuración verticillus-spira. F: Configuración flexibilis. RA: Configuración retinaculum-apertum.

cultivables su ubicación es, principalmente, endoliquénica (Fig. 2). Es importante destacar que el número de actinomicetes en relación a las UFC x g-1 totales es el mismo en el interior del talo de ambos líquenes, aproximadamente $1 \%$.

De las UFC $\mathrm{x} \mathrm{g}^{-1}$ totales provenientes de los talos de P. berberina, veintitrés (23) aislamientos correspondieron a actinomicetes cultivables, de los cuales veintiún (21) son endoliquénicos y 2 ectoliquénicos. En base a sus características morfológicas y fisiológicas, diecisiete (17) cepas se identificaron como Actinoplanes (2), Dactylosporangium (1), aff. Pseudonocardia (1), Micromonospora (6), aff. Micromonospora (1), Streptomyces (5), Streptosporangoium (1) y seis (6) no pudieron asignarse, todavía, a un taxón conocido de actinomicetes. Las características morfológicas y fisiológicas (ej. presencia o ausencia y color del micelio de sustrato y aéreo, tipo de

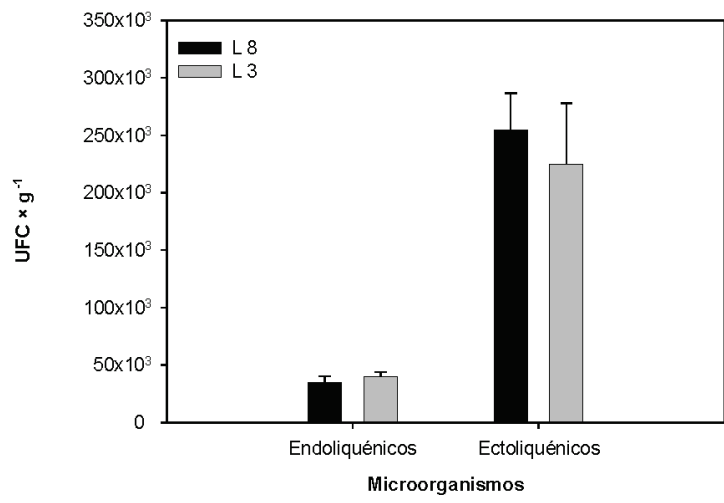

Fig. 1. Número total de unidades formadoras de colonias de microorganismos aisladas de $P$. berberina por gramo de liquen (UFC $\times$ $\mathrm{g}^{-1}$ ). Microorganismos aislados de la superficie (microorganismos ectoliquénicos) y del interior del talo (microorganismos endoliquénicos). L3 = Liquen 3; L8 = Liquen 8. Los datos representan a la media \pm desvío estándar (software Statistica 7.0). 
esporas, producción de pigmentos en el medio de cultivo) de cada una de ellas se indican en la Tabla 1. En la Figura 3 se muestran algunas de las cepas más representativas de los aislamientos de actinomicetes cultivables asociados a $P$. berberina. Estudios moleculares futuros completarán estas identificaciones.

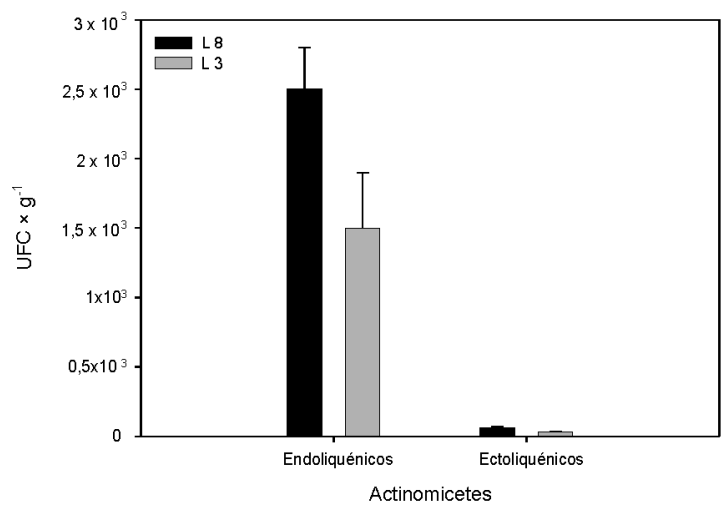

Fig. 2. Número de unidades formadoras de colonias de actinomicetes por gramo de $P$. berberina (UFC $\left.\times \mathrm{g}^{-1}\right)$. Actinomicetes aislados de la superficie del talo = ectoliquénicos; actinomicetes aislados del interior del talo: endoliquénicos. L3 = Liquen 3; L8 = Liquen 8. Los datos representan a la media \pm desvío estándar (software Statistica 7.0).
El número de actinobacterias cultivables, aisladas de ambos líquenes, concuerda con los datos presentados por González et al. (2005) para el caso de especies saxícolas y arborícolas de Alaska, Hawai e Islas Reunión. En base a esta coincidencia, el factor limitante para el establecimiento de las poblaciones de actinomicetes podría resultar del control fisiológico por parte del hongo liquenizado, de la disponibilidad espacial del liquen, o bien, como lo sugieren Grube \& Berg (2009), del ambiente donde se encuentra el microescosistema liquénico.

Aunque no fue objeto de este trabajo establecer la ubicación de estos microorganismos respecto a la anatomía interna del talo, estudios futuros sobre su disposición espacial dentro del liquen darán una idea más precisa sobre la relación simbiótica entre ambos.

A partir de los datos obtenidos resultará valioso determinar si existe algún tipo de patrón en su distribución, ubicación espacial y selección de su huésped por parte de los actinomicetes. Los líquenes, vistos como microecosistemas, impulsan nuevos interrogantes sobre la estabilidad y variación de los microorganismos asociados al holobionte. La presencia de comunidades de bacterias, y más específicamente de actinomicetes, en los hongos liquenizados abre nuevas expectativas a su estudio funcional en la simbiosis liquénica.
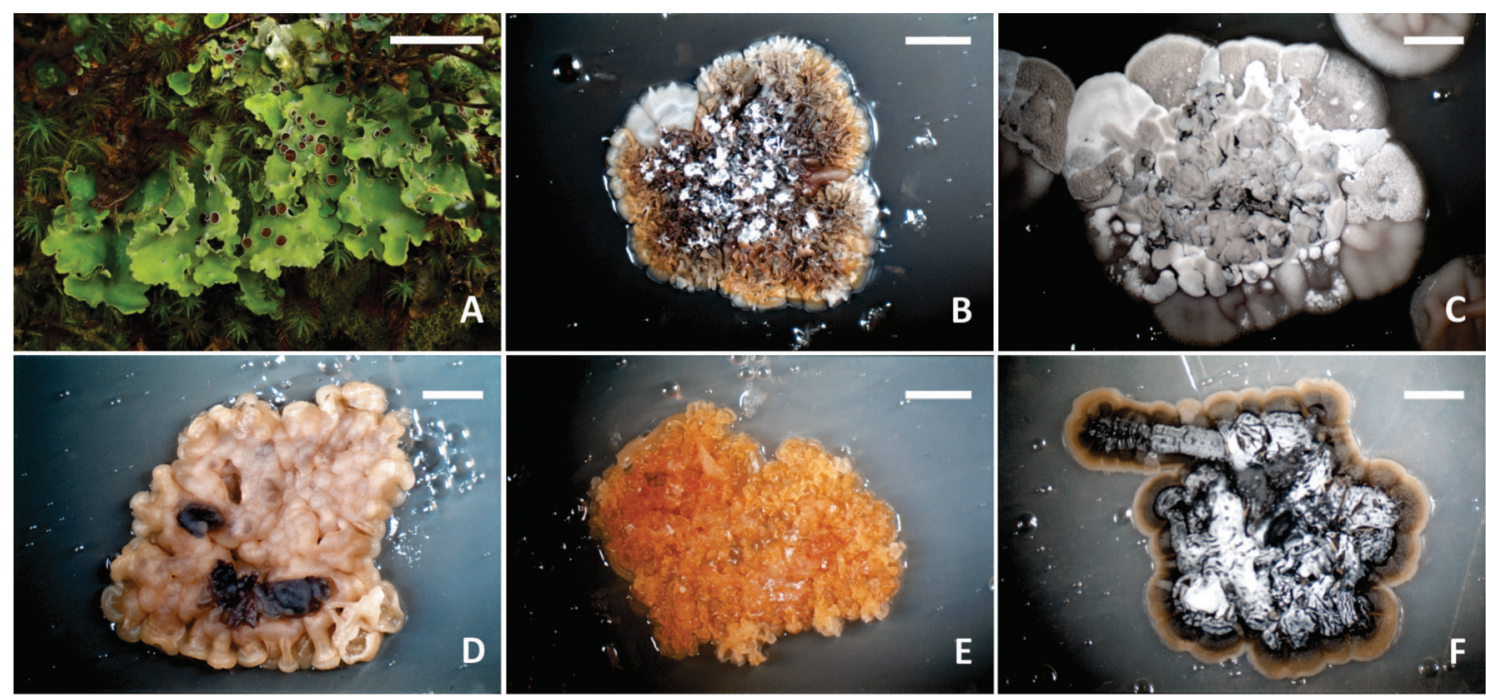

Fig. 3. Actinomicetes cultivables asociados a Pseudocyphellaria berberina. A: Pseudocyphellaria berberina (BCRU). B: Streptomyces sp. (BCRU MITI 5). C: Streptomyces sp. (BCRU MITI 15). D: aff. Micromonospora sp. (MITI BCRU 1 ${ }^{\text {b }}$ ). E: Micromonospora sp. (BCRU MITI 2). F: Micromonospora sp. (BCRU MITI 3). Escala: $\mathrm{A}=5 \mathrm{~cm} ; \mathrm{B}-\mathrm{F}=0,5 \mathrm{~cm}$. 


\section{Agradecimientos}

Agradecemos a la Secretaría de Investigación de la Universidad Nacional del Comahue (UNComahue), al Consejo Nacional de Investigaciones Científicas y Técnicas (CONICET) y a la Agencia Nacional de Promoción Científica y Tecnológica (ANPCyT) por el apoyo financiero brindado.

\section{Biblografía}

BATES, S. T., G. W. G. CROPSEY, J. G. CAPORASO, R. KNIGHT \& N. FIERER. 2011. Bacterial communities associated with the lichen symbiosis. Environ. Microbiol. 77: 1309-1314.

CABRERA, A. L. \& A. WILLINK. 1973. Biogeografia de América Latina. Monografía 13. Serie de Biología. Secretaría General de la Organización de los Estados Americanos, Washington DC.

CARDINALE, M., A. M. PUGLIA \& M. GRUBE. 2006. Molecular analysis of lichen-associated bacterial communities. FEMS Microbiol. Ecol. 57: 484-495.

CARDINALE, M., J. VIEIRA DE CASTO JR., H. MÜLLER, G. BERG \& M: GRUBE. 2008. In situ analysis of the bacterial community associated with the reindeer lichen Cladonia arbuscula reveals predominance of Alphaproteobacteria. FEMS Microbiol. Ecol. 66: 63-71.

CARDINALE, M., J. STEINOVÁ, J. RABENSTEINER, G. BERG \& M. GRUBE. 2012. Age, sun and substrate: triggers of bacterial communities in lichens. Environ. Microbiol. Rep. 4: 23-28.

DA SILVA, N. M. V., T. M. PEREIRA, S. A. FILHO \& T. MATSUURA. 2011. Taxonomic characterization and antimicrobial activity of actinomycetes associated with foliose lichens from the Amazonian ecosystems. Aust. J. Basic Appl. Sci. 5: 910-918.

GONZÁLEZ, I., A. AYUSO-SACIDO, A. ANDERSON \& O. GENILLOUD. 2005. Actinomycetes isolated from lichens: evaluation of their diversity and detection of biosynthetic gene sequences. FEMS Microbiol. Ecol. 54: 401-415.

GOODFELLOW, M. 2012. Phylum XXVI.Actinobacteria phyl. nov. En: GOODFELLOW, M., P. KÄMPFER, H.-J. BUSSE, M. E. TRUJILLO, K. SUZUKI, W. LUDWIG \& W. B. WHITMAN (eds.), Bergey's Manual of Systematic Bacteriology: 2nd ed., Vol. 5 (The Actinobacteria), pp. 33-34. Springer, New York.
GRUBE, M. \& G. BERG. 2009. Microbial consortia of bacteria and fungi with focus on the lichen symbiosis. Fungal Biol. Rev. 23: 72-85.

GRUBE, M., M. CARDINALE, J. VIEIRA DE CASTRO, H. MÜLLER \& G. BERG. 2009. Species-specific structural and functional diversity of bacterial communities in lichen symbioses. ISME J. 3: 11051115.

HODKINSON, B. P. \& F. LUTZONI. 2009. A microbiotic survey of lichen-associated bacteria reveals a new lineage from the Rhizobiales. Symbiosis 49: 163180.

HODKINSON, B. P., N. R. GOTTEL \& C. W. SCHADT. 2012. Photoautotrophic symbiont and geography are major factors affecting highly structured and diverse bacterial communities in the lichen microbiome. Environ. Microbiol. 14: 147-161.

MÖLLER, E.-C. 1994. Diversität innerhalb antarktischer Pilzpopulationen und innerhalb der kosmopoliten antarktischer Pilzart Chaunopycnis alba W. Gams. Dissertation, Techn. Univ., Braunschweig.

MUSHEGIAN, A. A., C. N. PETERSON, C. C. M. BAKER \& A. PRINGLE. 2011. Bacterial diversity across individual lichens. Appl. Environ. Microbiol. 77: 4249-4252.

ROUSK, J. \& P. BENGTSON. 2014. Microbial regulation of global biogeochemical cycles. Front. Microbiol. 5: $1-3$.

SHIRLING, E. B. \& D. GOTTLIEB. 1966. Methods for characterization of Streptomyces species. Int. J. Syst. Bacteriol. 16: 313-340.

VOBIS, G. 1997. Morphology of Actinomycetes. En: MIYADOH, S., M. HAMADA, K. HOTTA, T. KUDO, A. SEINO, G. VOBIS \& A. YOKOTA (eds.). Atlas of Actinomycetes, pp. 180-191. Asakura, Tokio.

VOBIS, G. 2006. The genus Actinoplanes and related genera. En: DWORKIN, M.; S. FALKOW, E. ROSENBERG, K. -H. SCHLEIFER \& E. STACKEBRANDT (eds.), The Prokaryotes: A handbook on the biology of bacteria. Vol. 3: Archea and Bacteria: Firmicutes, Actinomycetes. 3rd ed., pp. 623-653. Springer, New York.

Recibido el 14 de mayo de 2014, aceptado el 10 de septiembre de 2014. 
